\title{
Gradhiva
}

GRADHIV

Revue d'anthropologie et d'histoire des arts

$14 \mid 2011$

Carl Einstein et les primitivismes

\section{Les trois moments de Carl Einstein}

Carl Einstein's Three Moments

Isabelle Kalinowski

\section{OpenEdition}

Journals

Édition électronique

URL : http://journals.openedition.org/gradhiva/2277

DOI : $10.4000 /$ gradhiva. 2277

ISSN : 1760-849X

\section{Éditeur}

Musée du quai Branly Jacques Chirac

\section{Édition imprimée}

Date de publication : 30 novembre 2011

Pagination : 100-121

ISBN : 978-2-35744-046-3

ISSN : 0764-8928

Référence électronique

Isabelle Kalinowski, « Les trois moments de Carl Einstein », Gradhiva [En ligne], 14 | 2011, mis en ligne le 30 novembre 2014, consulté le 01 mai 2019. URL : http://journals.openedition.org/gradhiva/2277 ; DOI : 10.4000/gradhiva.2277 


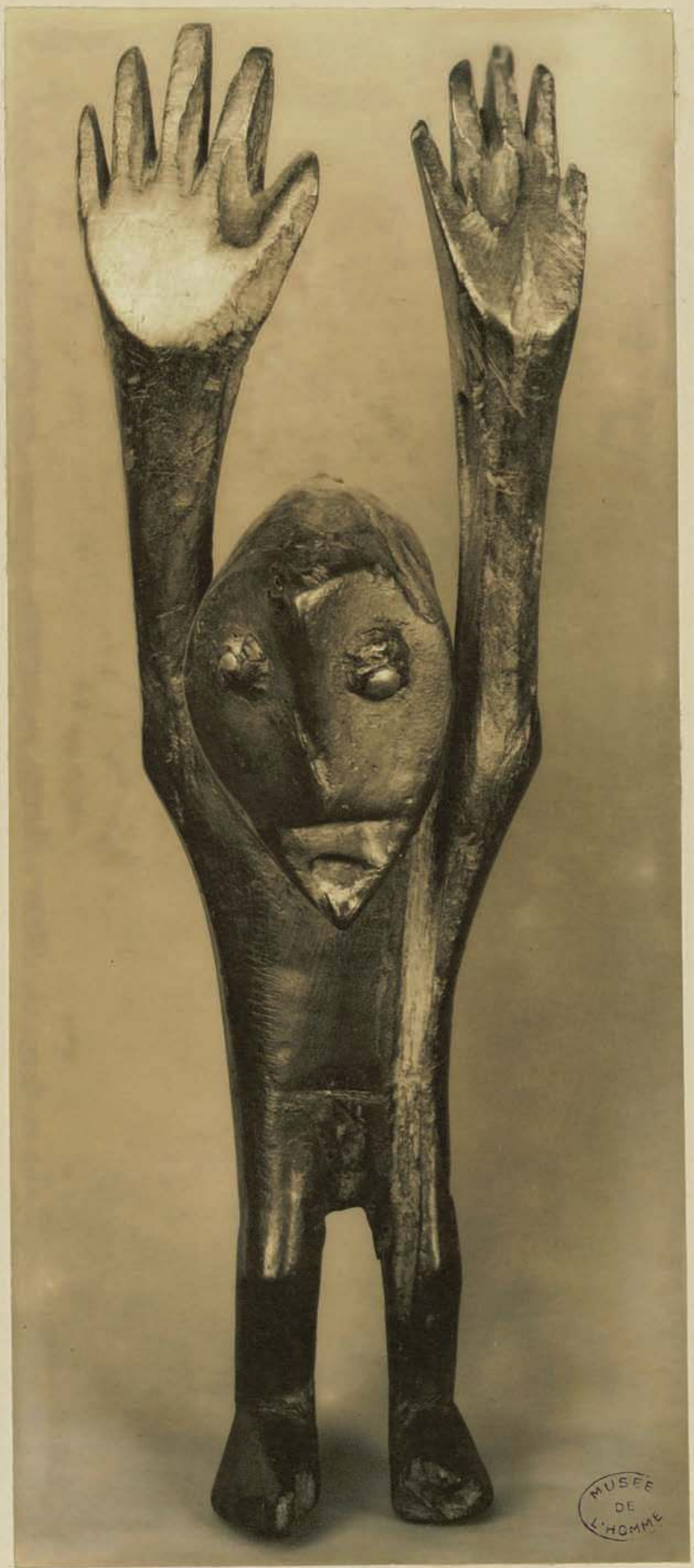




\section{Les trois moments ${ }^{\mathrm{de}}$ Carl Einstein}

Isabelle Kalinowski

Fig. 1 Walker Evans, Statuette, Congo belge, $n^{\circ} 397 \mathrm{du}$ portfolio African Negro Art, MoMA, 1935, musée du quai Branly @ Walker Evans Archive, The Metropolitan Museum of Art.
Texte de circonstance, rédigé en 1933 pour le catalogue d'une exposition newyorkaise, «Exposition de statuettes de bronze antiques antérieures à notre ère " ne fait pas partie des essais que la critique einsteinienne a privilégiés. Nous lui accorderons cependant une place de choix parce que, dépassant le cadre de l'archéologie méditerranéenne et orientale qui était celui de l'exposition, Carl Einstein y livre sous une forme peut-être plus accessible qu'ailleurs un modèle de pensée qui structure sa vision des cultures et des arts, et qui constitue une véritable clé pour la lecture de ses œuvres. Ce modèle, d'inspiration allemande et dans lequel on reconnaît sans peine, malgré ses vigoureux remaniements, un héritage post-hégélien, est une tripartition, un ensemble de trois moments qu'Einstein distingue dans la continuité d'une tradition mais en les réinterprétant de façon très personnelle. C'est à l'examen de ce modèle ternaire et à l'exploration de quelques-unes de ses implications qu'est consacré le présent article : son objectif n'est pas généalogique, car la part d'originalité de la tripartition einsteinienne l'emporte largement sur celle des influences subies; il s'agit de faciliter une entrée dans l'œuvre d'Einstein à partir des ouvertures qu'il ménage lui-même dans cet essai synthétique.

Dans "Exposition de statuettes de bronze antiques", ce sont moins les particularités stylistiques et morphologiques de la statuaire de la haute Antiquité orientale et méditerranéenne qui retiennent l'attention d'Einstein que la dynamique culturelle dans laquelle les œuvres ont vu le jour: un creuset d'échanges artistiques particulièrement denses, qui ont donné naissance à des formes hybrides. Einstein associe les pièces qu'il étudie à une temporalité particulière : des moments charnières situés entre le temps des pures migrations, ou des déplacements dans l'espace, et celui de l'installation durable de cultures "classiques ", comme la culture grecque ou la culture romaine. Il distingue ainsi trois moments : «nomade», «tectonique» et «classique». Les formes artistiques 
qu'il évoque appartiennent à des mondes urbains et sédentarisés, mais, selon ses propres termes, non encore «standardisés ». Dans ce texte, Einstein valorise les idoles cycladiques contre la Grèce du Parthénon, l'art étrusque contre l'art romain de l'époque impériale. Mais il ne prend pas pour point focal une authenticité des commencements dont il déplorerait la perte. Il construit, en se référant explicitement à Nietzsche, un modèle ternaire dont les "classicismes " n'occupent plus le centre et constituent même le moment de décadence. Cependant, l'acmé de cette tripartition ne réside pas non plus, pour lui, dans son moment premier, qu'il décrit comme une forme de nomadisme "chaotique ». Elle correspond plutôt à un art de "bâtisseurs » de cités, à la cohésion de collectivités neuves qui s'installent dans un lieu et y instaurent un ordre. La singularité de la tripartition einsteinienne, nous allons le voir, réside dans la valorisation de ce moment second, le moment "tectonique ", qui n'était généralement regardé que comme un moment de transition, rarement mis en avant dans les versions traditionnelles de ce modèle ternaire. Elle réside également dans la redéfinition des trois moments comme un ensemble cyclique et non plus comme une progression dialectique.

\section{Le modèle tripartite d'Einstein dans «Exposition de statuettes de bronze antiques »}

«L'homme angoissé par la mort s'efforce de préserver la vie dans l'effigie, et le sédentaire marqué par l'angoisse du chaos a recours aux formes tectoniques. La survie des défunts est liée à celle des formes corporelles; voilà pourquoi on aime la pierre, qui ne bouge pas." Le ton est donné : dans «Exposition de statuettes de bronze antiques", le moment "tectonique» prime sur les autres, avec les deux formes artistiques qui en sont les corrélats, l'architecture et la sculpture, et le matériau commun de ces deux arts, la pierre. Pourtant, bien qu'Einstein ait associé, dans ce texte comme dans bien d'autres, la sculpture à la sédentarité, à l'urbanité, à l'architecture et au matériau immobile entre tous, il a consacré la majeure partie de ses essais sur la sculpture à des œuvres potentiellement mobiles, des statuettes de bronze, ici, de bois, ailleurs, plutôt qu'à des effigies de pierre inamovibles. Il a invariablement privilégié la ronde-bosse "portative "; il ne s'intéresse guère aux basreliefs architecturaux. Il évoque volontiers le rapport organique entre sculpture et architecture, mais les formes plastiques qui retiennent son attention sont le plus souvent détachées de tout support bâti. Cette ambiguïté est constitutive de sa définition de la sculpture et du moment tectonique, qui n'est pas univoque et s'inscrit dans une polarité plutôt que dans l'assignation limitative à une position statique.

Dans le texte sur les bronzes antiques archaïques, Einstein cite plusieurs fois des inscriptions qui énoncent au subjonctif un désir de permanence qu'elles ne donnent pas pour réalisé. Marquée par la succession incessante de centres de pouvoir aussi grandioses que provisoires, l'histoire du Proche-Orient antique, qui constitue le référent du texte, trace l'horizon de cette tension, inhérente à la sculpture royale, entre survie éternelle et disparition. L'invention de l'écriture en Mésopotamie, qui n'est pas directement thématisée ici par Einstein, est en revanche implicitement associée par lui, avec beaucoup d'insistance, à cette fonction de la sculpture comme facteur de préservation potentielle. Einstein ne décrit pas d'œuvre précise mais cite de nombreux textes qui, le cas échéant, sont inscrits sur des statues. L'écriture, magique elle aussi, doit renforcer l'efficacité attendue de la statue, qui est par là simultanément affirmée et mise en 


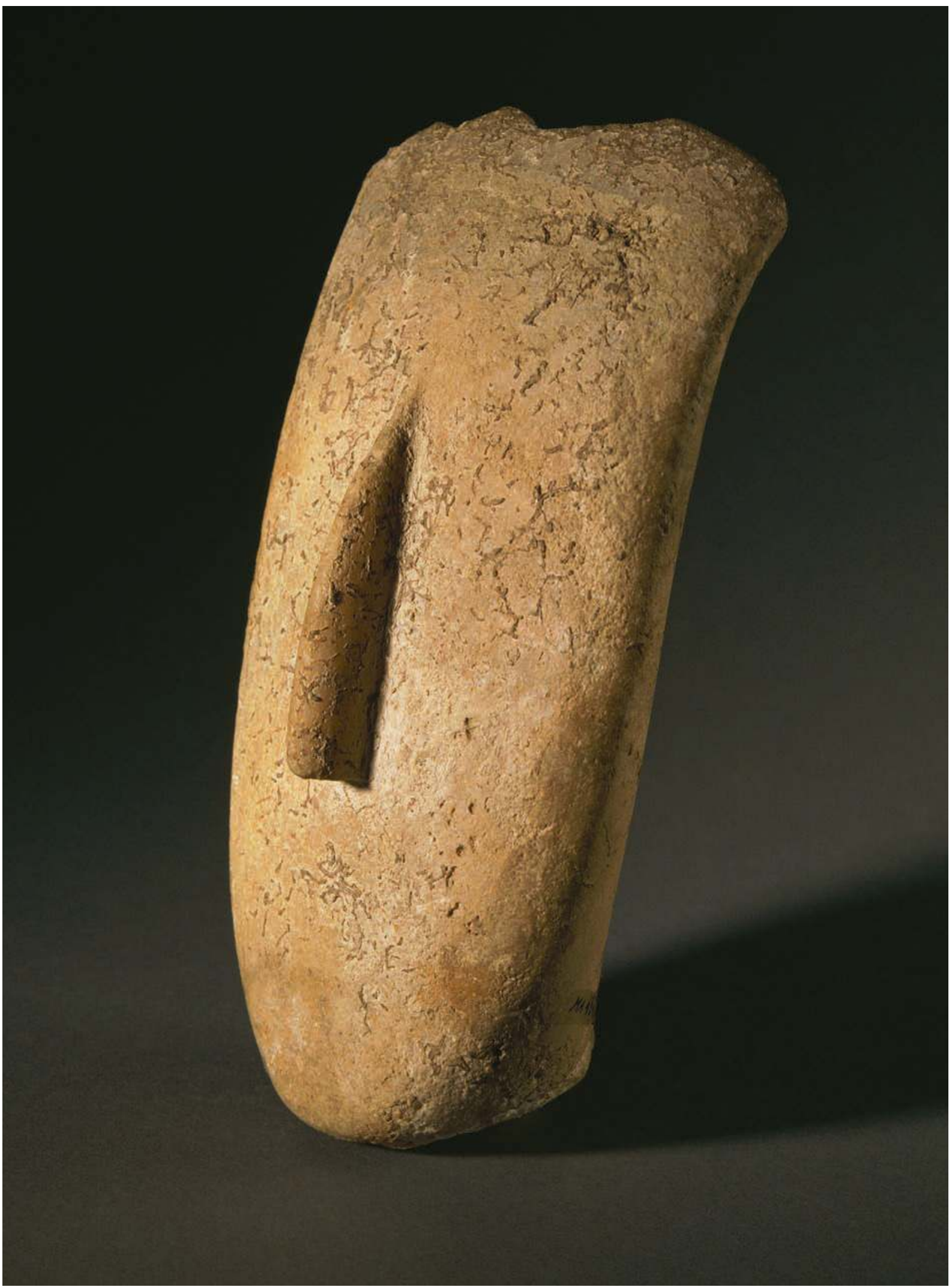

Fig. 2 Tête d'une idole cycladique du groupe de Spédos, 2700/2300 av. J.-C., Paris, musée du Louvre @ RMN / DR. 


\section{CARL EINSTEIN ET LES PRIMITIVISMES}

-

1. Sur cette question, voir PierreYves Le Pogam, La Sculpture à la lettre, Paris, Musée du Louvre Éditions, 2008.

2. Lettre de Carl Einstein à DanielHenry Kahnweiler, été 1938 (Einstein-Kahnweiler, 1993, p. 97).

3. Einstein, 1925, p. 198.

4. La notion de canon était au centre d'un article publié dans le premier numéro de Documents, en 1929 : « L'art sumérien : les conventions de la statuaire » de Georges Contenau. L'auteur défendait l'idée que ce qui pouvait être pris pour une maladresse artistique, le caractère extrêmement trapu des statues sumériennes assises, correspondait en réalité à un choix délibéré, la soumission à un canon de proportions bien défini. doute. Le style qu'Einstein examine le plus attentivement dans le texte est un style littéraire, le style hymnique qui vise à pérenniser la gloire du roi, et un lien est ainsi établi entre sculpture et écriture ${ }^{1}$. Une faute d'orthographe, dans une lettre d'Einstein à Daniel-Henry Kahnweiler, résume bien le propos : « couvrir du papier blanc avec de l'ancre ${ }^{2}$ ".

Dans ce contexte, il n'est pas surprenant qu'Einstein se soit intéressé à la figure du roi néosumérien Gudéa, prince de Lagash, qui se fit représenter en roi bâtisseur, comme dans la série de statues dites de «l'Architecte au plan ». Le roi, assis, porte sur ses genoux le dessin d'un temple dédié au dieu Ningirsu, gravé dans la pierre de sa statue, dont il a lui-même dessiné les plans tels qu'ils lui ont été dictés en rêve par le dieu en personne, avec le détail des rites de fondation à respecter. L'œuvre bâtie ou sculptée n'est pas seulement là pour honorer le dieu et se concilier ses bonnes grâces; le dieu, l'artiste-artisan et le roi (roi-dieu plutôt que roi-prêtre) tendent à ne faire plus qu'un dans le dessin de l'œuvre ou, comme le relève Einstein, dans l'écriture : «Gudéa de Tello affirme que les plans des [temples] qu'il a fait bâtir lui ont été révélés par les dieux en rêve. Sa déesse protectrice Nisaba [...] "tenait à la main le pur stylet d'écriture". » L'écriture, caution divine, devient l'agent d'une circulation affirmée comme parfaite entre hommes et dieux. Cependant, son efficacité est paradoxale : elle possède le pouvoir magique de contraindre les dieux parce qu'elle a reçu celui-ci des dieux eux-mêmes. Une définition très comparable de la magie avait déjà été proposée par Einstein dans un passage des Légendes africaines de 1925 : les hommes reçoivent de l'esprit même la statuette-fétiche qu'ils doivent reproduire à l'identique, par l'intermédiaire du magicien, pour se protéger contre l'action néfaste des esprits, et l'esprit leur révèle encore le détail des rites qui en assurent l'efficacité et la liste des «ingrédients » qui doivent y être placés.

Genèse du Mikisi Mihake

Un jour, Ngoy rendit visite à Nkulu, l'esprit. Il lui dit : «Grand esprit, ne vois-tu pas combien les hommes sont misérables? Maladies, guerres, famines tourmentent ces infortunés. Donne-moi un remède contre ces souffrances." Nkulu agréa à sa demande. Il alla chercher dans le fond du lac Kila, son séjour, une statuette longue comme le pouce, destinée à servir de modèle (de canon). « Ngoy, dit-il, je te donne là le remède infaillible contre les maux de toute sorte. Va voir les hommes et dis-leur de fabriquer des fétiches comme celui-ci. Puis apporte-les moi. » Ngoy exécuta ce commandement. Il appela Bwana Kilumba, le magicien, et lui enseigna à fabriquer des images comme celle-là. Le magicien en confectionna diverses imitations et les donna à Ngoy, qui les apporta à Nkulu.

Celui-ci enseigna la connaissance et les formules magiques pour placer les ingrédients dans les fétiches.

Le moment du «canon» est celui où, comme le dit Einstein dans le texte sur les bronzes antiques, «le rite des formes est répété avec humilité4 ». L'art, devenu " dogmatique », est alors "placé au service d'un ordre puissant et strict». Il s'inscrit dans un "cosmos stable » où le cours des affaires humaines est ajusté à celui des saisons et ce dernier à la trajectoire invariable des « astres ». Il est dominé par l'architecture, art de la sédentarité, y compris celle des dieux qui «sont devenus sédentaires et résident dans les temples ». Pourtant, ce moment de concordance généralisée, de triomphe de l'identité, de l'imitation, de la répétition, est aussi celui de l'invention du symbole qui porte en lui le principe de différenciation. Le symbole renvoie au dieu mais bat en brèche son «identité morte » en lui donnant accès à la métamorphose. Simultanément, il démultiplie ainsi la puissance de la divinité et la circonscrit : 
Dans l'apostrophe au dieu, on accumulait et additionnait les métaphores; le dieu se métamorphosait de symbole en symbole et, dans le symbole, il faisait la preuve de son efficacité sans limite sur les astres, les animaux, la terre et la mer. Les symboles recelaient le cosmos des métamorphoses du dieu et du souverain; et peut-être cette symbolique fut-elle le premier jeu de masques, la forme ancienne de la fuite devant l'identité morte.

Dans le symbole, on évite de nommer les noms divins, ces doubles que l'on redoute, et on a recours à la métaphore protectrice. Dans la symbolique, on accroît la réalité des dieux en enrichissant de forces et d'objets nouveaux leur sphère magique et en élargissant celle-ci. Le dieu révèle sa puissance active dans le drame des métamorphoses.

La notion, chère à Einstein, de "drame des métamorphoses " revient plusieurs fois dans le texte. Elle fait le lien entre les deux premiers moments de la tripartition, le nomadisme et l'«art urbain tectonique ", entre lesquels s'établissent des circulations. Le troisième moment, celui du classicisme, est quant à lui placé à part; Einstein l'assimilait à une forme de déclin ou, selon ses propres termes, de "standardisation " et de "mécanisation». Ces trois moments ne décrivent qu'une séquence d'évolution partielle, repérable à certaines époques et dans certains lieux, et non un schéma évolutionniste : «Rien ne me paraît plus naïf que la croyance selon laquelle tous les courants d'une culture suivent un cours unifié ", rappelaitil dans ce texte. Il n'entendait aucunement poser une série chronologique invariable mais distinguer, pour l'analyse, trois grandes tendances qui pouvaient se conjuguer dans la réalité. Il valorisait spécifiquement la deuxième et la décrivait comme capable de recueillir, sous une forme pacifiée et assimilable, les acquis de la première : ainsi, les métamorphoses infinies qui accompagnent le "flot des visions " des "nomades, chasseurs et bergers qui se déplacent sans trêve " perdent leur caractère d' "hallucinations subjectives » dans l'art urbain tectonique, où elles acquièrent la "validité collective» du «symbole». Les «dynamismes » et "signes d'action " caractéristiques du nomadisme perdent, dans le tectonique, la "rapide caducité " qui les condamnait à se substituer indéfiniment les uns aux autres.

\begin{tabular}{|c|c|c|}
\hline NOMADISME & ART URBAIN TECTONIOUE & CLASSICISME \\
\hline \multirow[t]{2}{*}{ hallucinations subjectives } & symbole & concept \\
\hline & validité collective & rationalisme \\
\hline \multirow[t]{2}{*}{ flot des visions } & métaphore protectrice & calcul \\
\hline & canon & \\
\hline \multicolumn{2}{|c|}{ drame des métamorphoses } & standardisation \\
\hline \multirow[t]{3}{*}{ mobilité graphique } & architecture & philosophie \\
\hline & sculpture & \\
\hline & style hymnique & \\
\hline réalisme & antinaturalisme & anthropomorphisme \\
\hline animisme & art de bâtisseurs & académisme \\
\hline \multirow[t]{3}{*}{ art animalier } & et de colonisateurs & ornement décoratif \\
\hline & & paraphrase \\
\hline & & allégorie inoffensive \\
\hline bronze & pierre & stuc \\
\hline chaos des forces & ordre, cosmos stable & réalité mécanisée \\
\hline \multicolumn{3}{|l|}{ difficilement intelligibles } \\
\hline esprits élémentaires & dieux sédentarisés & dieux anthropomorphes \\
\hline
\end{tabular}


La singularité de la tripartition d'Einstein ne tenait guère au modèle luimême, qui, avec différentes variantes, était une figure de pensée relativement classique de la tradition germanique post-hégélienne; elle résidait bien davantage dans le refus de l'historien de l'art de valoriser le troisième moment aux dépens des deux autres. Le rejet de l'idéal de l'anthropomorphisme, exemplairement incarné dans l'art grec classique, était inattendu dans un tel contexte : le modèle tripartite était le plus souvent convoqué pour opposer à une forme de particularité contingente la "personnalité » du troisième moment, synthèse réussie du singulier et de l'universel. La tripartition tout entière trouvait généralement son point culminant dans ce dépassement abolissant l'opposition entre subjectif et objectif. Einstein, lui, prenait le modèle à rebours et modifiait son orientation initiale.

Une des versions savantes les plus célèbres de ce schéma ternaire avait été livrée, en 1896, par le philologue classique Hermann Usener dans Les Noms de dieux : à partir d'une analyse de listes de noms de dieux, notamment des répertoires de divinités latines, il avait établi le caractère récurrent d'une tripartition entre les « dieux de l'instant ", entités ponctuelles vénérées hic et nunc, les « dieux spécifiques ", définis par leur fonction (parmi lesquels il rangeait les saints chrétiens), et enfin les "dieux personnels", seuls dotés d'une véritable individualité. Dans sa sociologie de la religion, Max Weber se référait explicitement à ce modèle et le reprenait à son compte en substituant seulement le terme de "dieux fonctionnels » à celui de "dieux spécifiques ». L'antithèse entre "dieux fonctionnels " et "dieux personnels», selon lui, trouvait une illustration paradigmatique dans l'opposition de la religion romaine et de la religion grecque. L'antagonisme entre le caractère "abstrait» des divinités romaines (la spécification rationnelle de leurs compétences) et celui, plus "personnel», des divinités grecques, se traduisait historiquement, notait-il, dans le fait que la religion avait favorisé, dans les deux cas, le développement de deux sphères de réalité bien distinctes : le droit, à Rome, l'art, en Grèce. «Les compétences des numina romains étaient délimitées de façon incomparablement plus nette et univoque que celles des figures divines de la Grèce; en revanche, l'humanisation des divinités grecques et la définition plastique et visuelle leur conférant une véritable "personnalité" étaient incomparablement plus poussées que dans la religion romaine traditionnelles. " L'idée d'une affinité spécifique entre la sculpture grecque et la représentation des «dieux personnels » était déjà présente chez Usener, qui évoquait même l'hypothèse, à ses yeux erronée, selon laquelle les seconds seraient nés de leur figuration artistique dans la plastique grecque ${ }^{6}$.

\begin{tabular}{lll} 
DIEUX DE L'INSTANT & DIEUX SPÉCIFIQUES & DIEUX PERSONNELS \\
\hline «idoles 》 & divinités romaines & divinités grecques \\
«fétiches 》 & caractère abstrait & caractère «plastique \\
(Usener, Weber $)$ & et rationnel & et visuel 》 (Weber $)$
\end{tabular}

5. Max Weber, Sociologie de la religion, trad. et présentation d'Isabelle Kalinowski, Paris, Champs Flammarion, 2006, p. 96.

6. Voir Hermann Usener, Götternamen, Bonn, F. Cohen, 1896, p. 302. 
grecque cessait de représenter le parangon absolu de la plastique. Selon Einstein, la force et la pérennité des Grecs n'avaient pas trouvé leur source dans l'art, mais plutôt dans la philosophie. Ce qu'il interprétait comme une survalorisation illégitime de l'art grec constituait, pour lui, une véritable "falsification sociologique ", à en croire ses notes préparatoires (posthumes) à un Manuel de l'art. La "genèse de la qualification de classique » et la vision épurée du «miracle grec " présupposaient une "oblitération de l'aspect historique» et un déni de l'origine non individuelle des innovations artistiques : une "réinterprétation falsificatrice de l'œuvre collective comme prouesse héroïque d'un individu » - une focalisation sur des personnalités d'artistes qui trouvait son pendant dans la généralisation de l'anthropomorphisme des statues?.

À l'opposé d'une telle tendance, Einstein mettait l'accent sur le rôle, dans le renouvellement des arts, de "peuples méconnus et décriés " qui contribuaient à l'émergence de formes inédites. Son texte intitulé «L'art des nomades d'Asie centrale " brosse un portrait des peuples itinérants en inventeurs de synthèses artistiques éclectiques ou de styles de "transit» dans lesquels "les formes s'attirent et circulent comme les caravanes et les troupeaux". Les nomades n'étaient pas des créateurs ex nihilo, ils développaient plutôt un "art curieusement conservateur ", emportant au passage la mémoire de formes en voie de désaffection là où elles avaient vu le jour; mais leur capacité d'innovation résidait dans la mise en relation d'éléments qui ne s'étaient jamais rencontrés auparavant : «Un art populaire, trop peu pris en compte par les savants, traité avec un certain mépris parce qu'il se trouve hors de la zone cultivée, classique. Difficile à interpréter car les œuvres les plus récentes conservent encore un fonds ancien; les temps s'entrecroisent en elles presque au hasard; un art curieusement conservateur, qui ne reprend souvent les motifs importants que lorsqu'ils ont perdu leur force."

Ainsi, le modèle tripartite développé par Einstein dans «Exposition de statuettes de bronze antiques" revêtait une dimension non pas évolutionniste, mais cyclique : le moment nomade ne représentait pas un commencement absolu, il remplissait davantage une fonction de renouvellement des formes par leur déplacement et leur recomposition plurielle. Einstein illustrait exemplairement cette idée par le cas des Étrusques, ancien peuple nomade aux origines obscures, qui avaient transporté en Italie, dont ils furent les «véritables colonisateurs ", les motifs de lointains « classicismes » oubliés pour en produire, une fois sédentarisés, une synthèse inédite dont les apports avaient nourri «les impulsions fondamentales de la culture romaine " et dont l'héritage formait "les éléments les plus nobles" de celle-ci. La "tradition des Latins" passait "obstinément sous silence cet héritage majeur, avec une suffisance de parvenus ", mais les dimensions étrusques de l'art romain rattachaient celui-ci à la "vieille tectonique orientale». Le "conservatisme " éclectique des nomades qui, ajoutait Einstein, n'étaient pas pour autant «des collectionneurs", devenait, de la sorte, le moteur de nombreuses innovations artistiques réalisées par des cultures sédentaires. Les peuples itinérants, les «mille-pattes", portaient à la fois le mort et le vif, et détenaient surtout la clé de la transmutation de l'un dans l'autre. En cela, ils remplissaient une fonction un peu comparable à celle que, dans le texte sur le Cavalier bleu présenté dans ce numéro, Einstein attribuait à l'inconscient, qui «stocke les héritages oubliés" mais dans lequel "sommeillent les forces non encore adaptées » qui vont permettre d'arracher à la fixité ces vestiges de passé. 


\section{DOSSIER}

\section{CARL EINSTEIN ET LES PRIMITIVISMES}

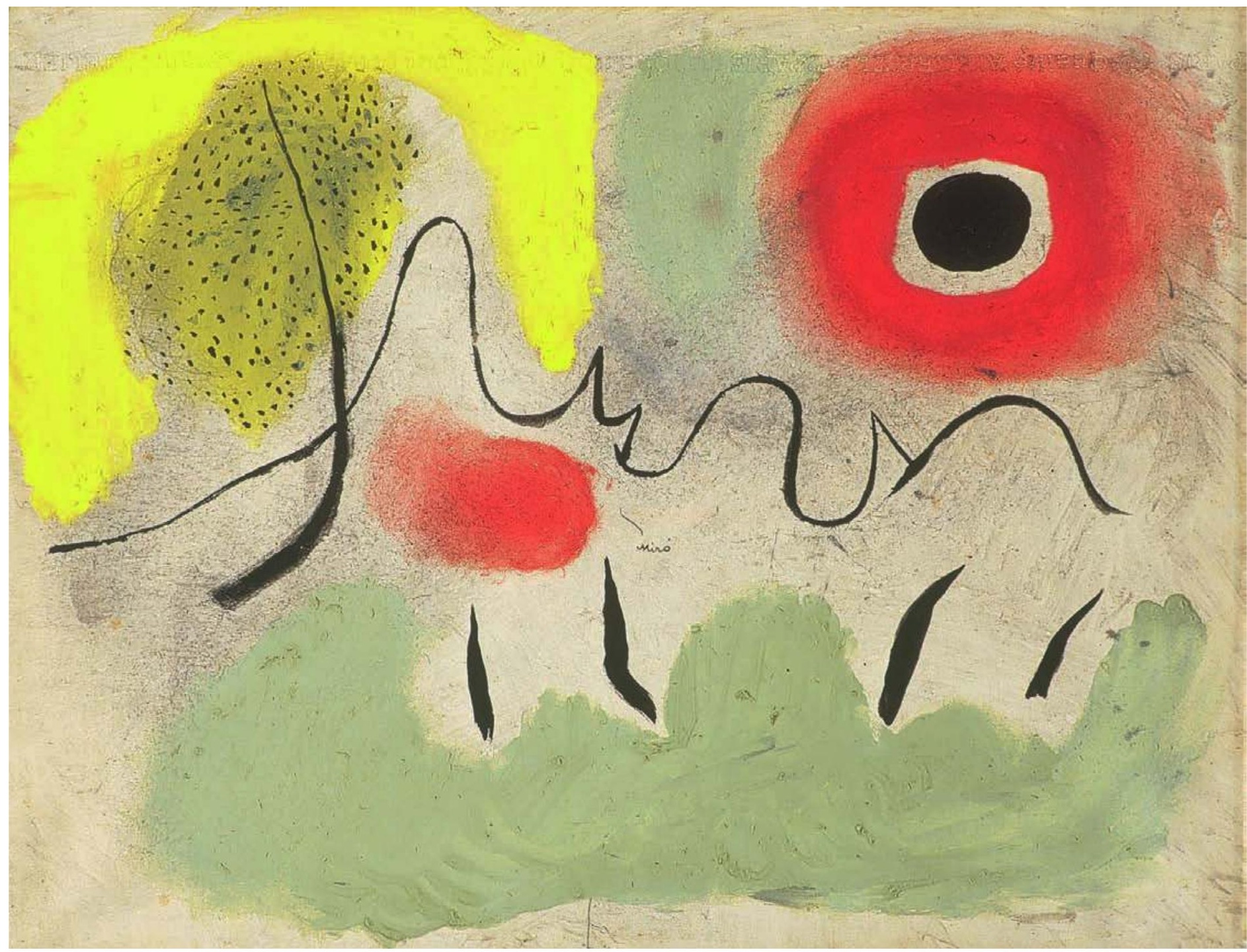

Fig. 3 Joan Miró, Souvenir de Montroig, 1937, collection particulière @ Successió Miró / ADAGP, Paris 2011. 


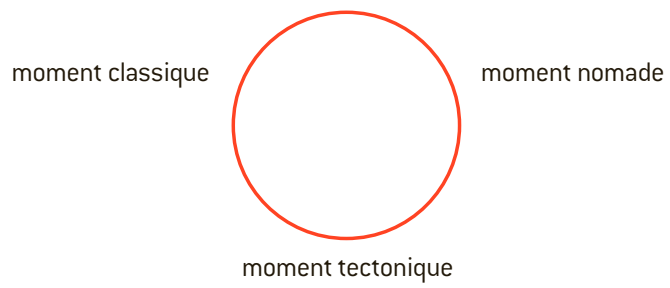

Par suite, une représentation graphique adéquate des trois moments einsteiniens doit rendre compte de leur caractère cyclique; au sens strict, on ne devrait pas parler d'un "premier", d'un "deuxième" et d'un "troisième " moment: Einstein lui-même s'abstenait de le faire. Par son aspect cyclique, la tripartition s'apparente aux cosmologies anciennes des sociétés archaïques; mais un fossé la sépare cependant de ces dernières. À aucun moment, en effet, elle ne trouve un ancrage dans les cycles de la nature, l'alternance des saisons et une succession de morts et de renaissances. Le moment nomade est le seul qu'Einstein rattache à un élément naturel : la proximité avec le monde animal, qui se traduit dans la thématique des objets d'art. Toute référence à la végétation est en revanche absente de son modèle ternaire, détaché d'un quelconque support organique. Einstein n'accorde guère d'attention, par exemple, au motif de la renaissance des plantes à la suite des crues ou du retour des pluies, si central dans l'art égyptien et l'art mésopotamien : chez lui, pas de rameaux verdissants, pas de "vases globulaires aux eaux jaillissantes", comme sur les sceaux-cylindres, les basreliefs et les fresques du Proche-Orient. La «métamorphose " au sens d'Einstein ne renvoie pas aux vestiges d'une philosophie de la nature, à la différence du modèle fameux de la «métamorphose des plantes» de Goethe : elle ne consiste en aucun cas dans un déploiement du même, dans l'éclosion de fleurs et de fruits déjà contenus dans un "germe " premier. Elle présuppose une confrontation productive avec l'altérité. C'est parce qu'un tel «événement » a lieu, rompant le toujours-déjà-là du temps mythique, qu'Einstein a recours à la notion de «drame des métamorphoses ». Le modèle ternaire einsteinien ne reconstitue pas une pensée mythique.

En définissant sa tripartition comme cyclique, Einstein rompait avec le modèle de la dialectique hégélienne; mais il ne remettait pas en cause un présupposé fondamental de celle-ci, incompatible avec une forme quelconque d'évolutionnisme : le fait que la dynamique des changements historiques était portée par la contradiction. C'est cette force contradictoire, dont la tension, parvenue à un point extrême, était capable de susciter de brusques renversements, qui était le moteur de l'élaboration du nouveau, et non une forme d'épanouissement linéaire et continu. Einstein avait fait sien cet héritage hégélien revisité par Karl Marx, et il ne renia à aucun moment cette pensée de l'antagonisme créateur.

Le modèle d'Einstein fut aussi marqué par l'impact d'historiens de l'art qui, tel Aloïs Riegl, avaient construit avant lui des formes rigoureuses d'" anti-classicisme ». Son apport singulier résida dans une définition fine et particulièrement nuancée de l'alternance entre les moments «classiques» et non classiques. La critique de l'hégémonie des classicismes pouvait en effet conduire à une revalorisation parfois un peu mécanique et idéalisante des "hybridations" stylistiques; l'insistance avec laquelle Einstein défendit l'importance du moment "tectonique" attestait son souci d'échapper à toute formulation trop schématique d'une alternative entre «classique » et «nomade». 
«Dominer les hallucinations » : angoisses du moment nomade

Le moment nomade, moteur premier de toutes les innovations, on l'a vu, est en même temps porteur d'une incomplétude qu'Einstein a associée, selon les textes, à différentes modalités d'un manque appelé à être surmonté dans le "tectonique ». Dans "L'art des nomades d'Asie centrale », il évoque non sans tendresse la douleur associée à l'errance sans fin en observant que celle-ci est inscrite dans la peau des visages, comme une balafre, sur les têtes de métal sculptées par les nomades : "Ces têtes sont saturées d'images qui suggèrent des voyages sans fin. Les joues noircies sont comme parcourues de chemins. " Il remarque également sur ces joues la présence du motif animalier qu'il appelle le «mille-pattes». Le sens de cette douleur, tel qu'Einstein l'explicite dans d'autres textes, est le danger d'être exposé à un excès de signaux extérieurs reçus sur un mode de trop grande passivité. Une des dernières lettres d'Einstein à Kahnweiler, datée du 6 janvier 1939 (et écrite en français), explicitait sans détours l'angoisse qu'il cherchait ainsi à conjurer, y compris en abandonnant ses manuscrits pour l'activisme du partisan de la guerre d'Espagne :

Quand j'ai quitté Paris sans dire un mot, je savais très bien pourquoi. [...] Je laissais mes contrats, une maison d'édition que l'on était en train de monter et pas mal des choses, et je suis content d'avoir fait cela, je m'en allais quand ça devait commencer d'aller très bien pour moi, mes manuscrits, ça dort; [mais j'ai voulu] sortir des mots, quitter les métaphores, car jamais je n'étais un rond-de-cuir poétique et jamais je le serai. Quand je rentrerai je ferai des bouquins solides, loin de tous les penchants des modernes et des bien-pensants de tous les avant-gardes, des livres durs et comiques, [...] d'ailleurs dans les temps qui courent le fusil est nécessaire pour compenser la lâcheté du stylo, ces littérateurs, nom d'un chien, ils écrivent des choses indifférentes parce qu'ils ne sont pas des hommes, mais les dupes des métaphores, les Flauberts des images, il ne s'agit pas seulement de se faire dominer par le poétique (masochisme littéraire), mais dominer les hallucinations, les mettre en rang, les utiliser. (EinsteinKahnweiler, 1993 : 106)

Lelittérateur " dévoré de métaphores comme onl'est de poux " (Gustave Flaubert) représentait donc une autre figure de l'incomplétude «nomade » à laquelle Einstein opposait la virilité de l'acte militant. Si l'on applique au passage qui vient d'être cité le schéma tripartite du texte sur les bronzes antiques, on constate qu'Einstein privilégiait une nouvelle fois le moment «solide » et « dur » où hommes et métaphores étaient «mis en rang ". Les deux autres moments, la phase «nomade » d'abandon aux «images» et "hallucinations» et la phase "mécanisée » des "ronds-de-cuir poétiques", se rejoignaient ici dans l'évocation des avant-gardes parisiennes de l'entre-deux-guerres. Celles-ci étaient opposées sur un mode dualiste à l'Espagne de la lutte républicaine. La tonalité martiale de ce passage ne doit cependant pas dissimuler qu'Einstein n'opposait pas frontalement art et combat, moins encore littérature et action. Quand il imaginait la vie qu'il pourrait mener après la guerre, il exprimait le vœu de s'installer à la campagne et, délaissant la critique d'art, de retourner à l'écriture littéraire de ses débuts : «Le seul service que je peux rendre après cette guerre, c'est d'essayer d'écrire de la bonne prose.» (Ibid. : 97) Il priait Kahnweiler de lui expédier des colis contenant des paquets de tabac et des classiques : «Voyez, Heini, je trouve ma petite vérité dans les vieux chinois, en Héraclite ou dans le sacrifice fou de Hoelderlin. Je ne change pas, je mûris, mais plus je deviens vieux, plus je deviens inquiet. [...] Avez-vous une petite éthique de Spinoza et les fragments de Novalis pour moi? » (Ibid. : 108) 


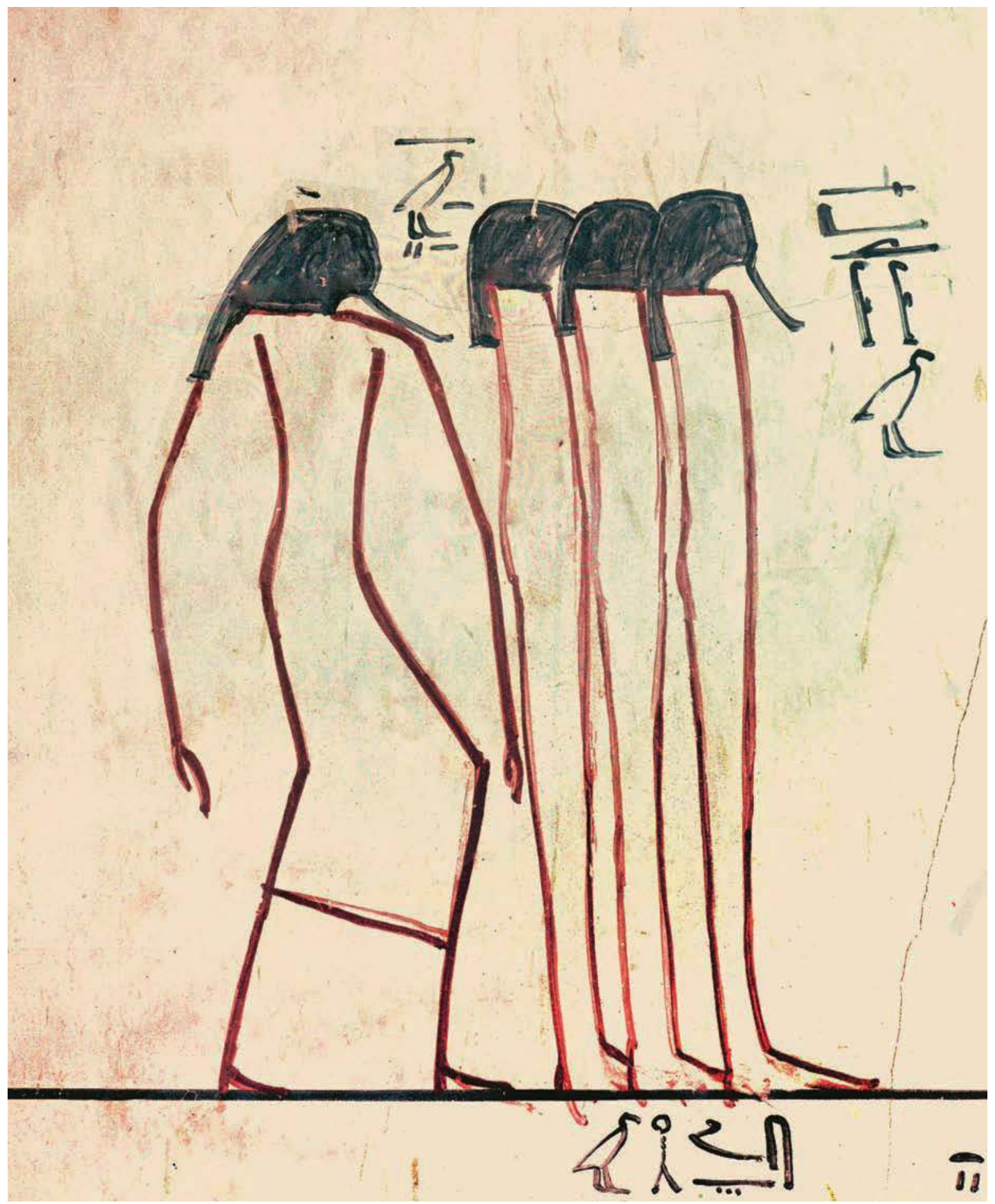

Fig. 4 Fresque de la tombe de Thoutmosis III avec une scène de Le Livre de ce qu'ily a dans l'Hadès, VIII dynastie, 1490-1440 av. J.-C. Werner Forman Archive. 


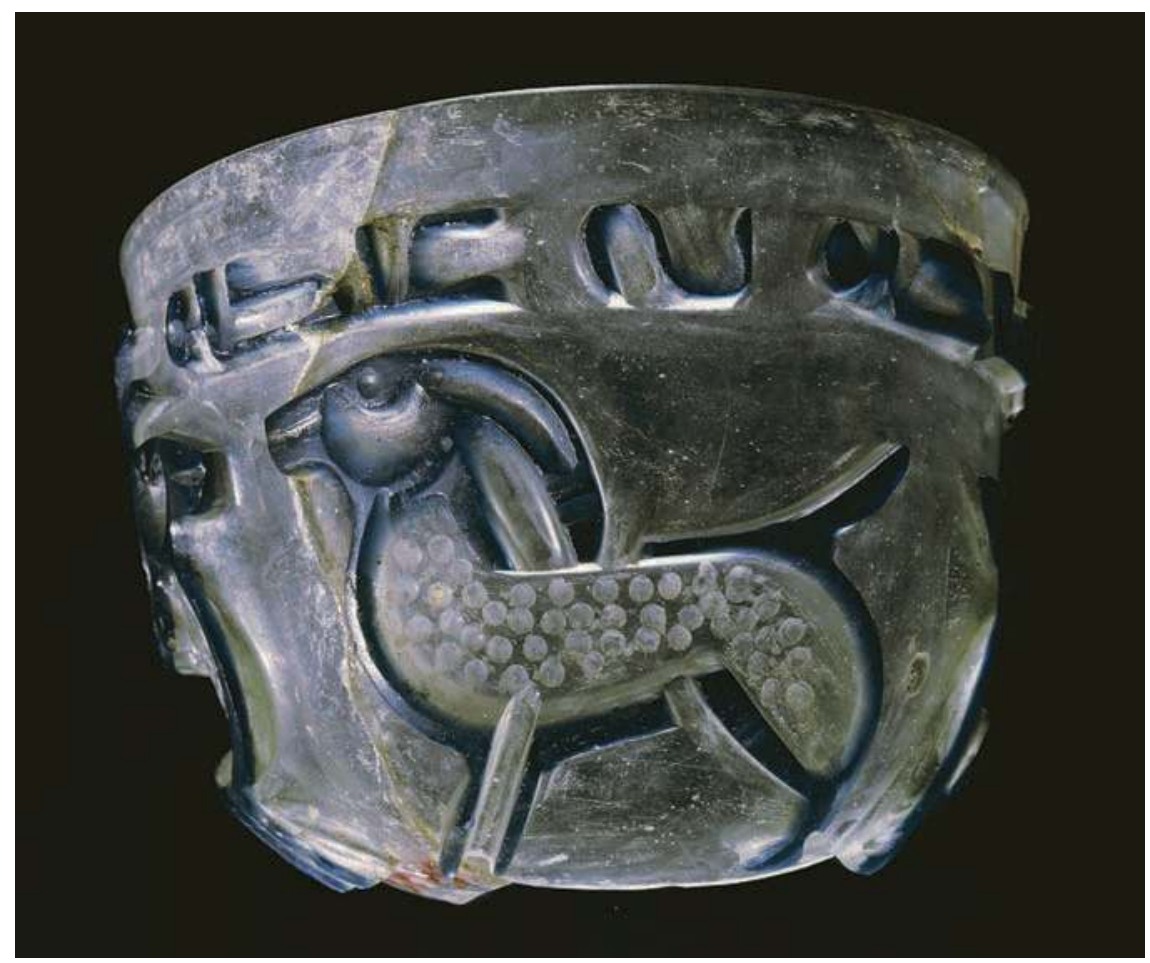

Fig. 5 Bol en verre, dont le relief représente des chèvres sauvages et porte une inscription coufique votive, $v^{e}{ }^{e}-x^{e}$ siècle, musée d'Art islamique, Le Caire. Werner Forman Archive.

L'alternative rigide de l'écriture et de l'action politique dont la vie d'Einstein offrit le modèle n'était donc pas conçue par lui comme absolue. Il n'en demeure pas moins que le moment du collectif, exemplairement incarné dans la « force de camaraderie » des combattants républicains, équivalent existentiel du moment tectonique dans l'histoire des arts, était regardé par lui comme l'unique réponse possible à une angoisse que l'écriture et, de façon générale, la création artistique éveillaient chez lui. Un des textes où Einstein a décrit le plus minutieusement la menace qui lui semblait en émaner, en l'occurrence le danger d'une passivité de l'artiste à l'égard de ses "hallucinations », est le long passage qu'il a consacré à Vassily Kandinsky dans le chapitre de L'Art du xx siècle dédié au Cavalier bleu. L'art visionnaire promu par ce groupe de peintres avait bousculé l'ordre figé de la mimèsis et c'était là, pour Einstein, un apport décisif, qu'il saluait en tant que tel : « Le peintre n'était plus là pour représenter ni pour arranger. » Il était devenu "un pur médium des visions », capable de bousculer "l'inertie statique des toiles classiques » et de "retranscrire de façon plus désinhibée des processus jusque-là demeurés à l'état inconscient ». Dans les termes du modèle tripartite, la peinture du Cavalier bleu correspondait ainsi à un moment «nomade» où s'ouvraient les portes de la vie psychique auparavant verrouillées par un classicisme statique, et qui inaugurait des déplacements libérateurs. «Kandinsky fut à l'origine d'une révolte emblématique d'une génération : la rupture avec les choses, le désir de peindre un événement psychique dans son immédiateté et sa nudité. » Cependant, l'expansion visionnaire de la peinture recelait le danger d'un nouvel enfermement : elle courait le risque de "rester prise dans un lyrisme subjectif». Le «retour au moi» avait alors "pour prix la perte du monde vu»: «Les choses 
étaient pulvérisées sous l'effet d'un pur solipsisme optique : ruine de l'objet. " Ainsi menacé par «la solitude de l'arbitraire», le peintre «s'épuisait » dans l'invention d'une "mystique dévoreuse de la forme». De ses visions, il n'était pas en mesure de tirer des "objets ", c'est-à-dire des formes ou des symboles dotés d'une validité collective. Il retombait ainsi dans "l'ornement » et le « décoratif ", autrement dit dans les mêmes ornières que le "classicisme » qu'il avait d'abord vilipendé. Tout comme dans la lettre à Kahnweiler où il critiquait les avant-gardes parisiennes, à la fois trop dominées par leurs «hallucinations » et trop installées, Einstein insistait ici sur la proximité entre les premier et troisième moments de la tripartition, lorsque le passage au "tectonique" s'avérait impossible. Quelles étaient les raisons d'un tel échec? Pourquoi ce passage n'avait-il pu s'effectuer?

Einstein expliquait cette impasse en constatant le fossé qui séparait les «primitivistes " modernes et les " primitifs " : «Les primitifs vivaient dans un monde spirituel dont la forme était préétablie. Les peintres comme Marc et Kandinsky voulaient, eux, tirer un tel monde de l'immédiateté du moi et s'épuisaient d'emblée dans le prologue spirituel. Ils ne disposaient pas d'un univers religieux structuré, d'un monde ordonné de symboles, et ils s'enfermaient ainsi dans une mystique égocentrique.» En soulignant cette différence, Einstein n'entendait cependant en aucun cas énoncer la nostalgie d'un moment révolu ni le désir d'un retour en arrière, pas plus que l'évocation du «cosmos stable» de certaines sociétés anciennes de la Méditerranée orientale, dans le texte sur les bronzes antiques, n'appelait une interprétation réactionnaire. Le moment tectonique ne pouvait, à proprement parler, faire l'objet d'aucune restauration. Einstein rejoignait en ce sens point par point le diagnostic de Weber à la fin de sa fameuse conférence de 1917 sur la science : «Jamais une nouvelle prophétie n'a vu le jour [...] pour la seule raison que beaucoup d'intellectuels modernes ressentent le besoin de meubler leur âme, pour ainsi dire, de vieilleries garanties authentiques, et se rappellent soudain, à cette occasion, que la religion qu'ils n'ont plus en a aussi fait partie; ni parce qu'ils s'aménagent alors, en guise d'ersatz, une sorte de chapelle domestique éclectiquement décorée de petits portraits de saints de tous les pays, ou bien se créent une compensation à travers toutes sortes d'expériences vécues auxquelles ils attribuent la dignité d'une possession mystique de la sainteté et qu'ils vont... vendre à la criée sur le marché du livre ${ }^{8}$.»

La double conséquence que Weber tirait d'un tel constat, à la fois du point de vue de l'histoire de l'art et du point de vue de la définition d'un sentiment collectif, recoupait également les vues d'Einstein : l'époque ne se prêtait plus à la conception d'un "art monumental " qui tendait, comme dans les réalisations de l'architecture berlinoise du début du xx siècle, vers le "monstrueux", elle exigeait au contraire un "art intime"; de la même façon, la seule "communauté " susceptible d'être instaurée dans un " acte de fraternité authentique » était désormais non plus un État, une entité politique à grande échelle, mais un groupe restreint en situation de rupture avec l'ordre politique dominant, comme les cercles de jeunes pacifistes avec lesquels Weber débattait des nuits durant :

Ce n'est pas un hasard si notre art le plus abouti est aujourd'hui un art intime et non monumental; ce n'est pas un hasard non plus si, aujourd'hui, c'est seulement au sein des cercles communautaires les plus restreints, entre des individus, en pianissimo, qu'on sent vibrer ce quelque chose qui rappelle les temps anciens où le pneuma prophétique embrasait les grandes communautés comme une vague de flammes et fondait leur unité. Si nous tentons à toute force de produire et d'«inventer » un nouveau sens de l'art monumental, nous n'obtenons que des créations difformes et
8. Max Weber, La Science, profession et vocation, trad. I. Kalinowski, Marseille, Agone, 2005, p. 55. 


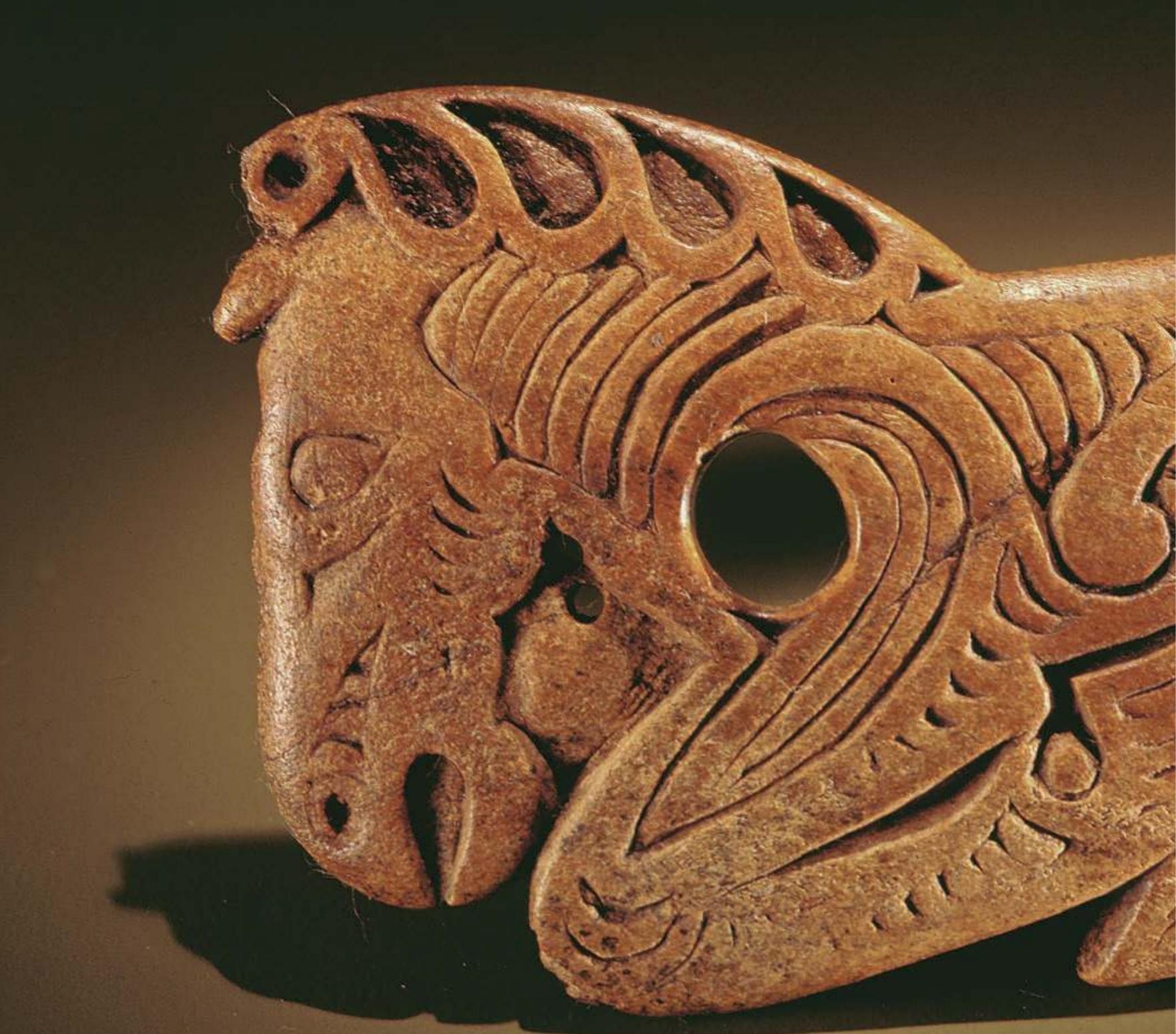

Fig. 6 Plaque en forme de cheval, nomades de l'Altai, ve-III siècle av. J.-C., musée de l’Ermitage, Saint-Pétersbourg @ Boltin Picture Library.

lamentables : songeons à tous les monuments de ces deux dernières décennies. De même, si nous tentons d'imaginer de nouvelles formes de religion sans être portés par une prophétie nouvelle et authentique, nous aboutissons à de semblables difformités, intérieures cette fois ${ }^{9}$.

Cette série de renversements, cependant, était loin d'aller de soi. Comment "l'art intime» et les petites "fraternités» en étaient-ils venus à remplir la fonction autrefois assumée par «l'art monumental » et les "grandes communautés »? Comment, pour Einstein, un «art de la révolte " pouvait-il se substituer au canon absolu de l'art "tectonique » et à son "univers religieux structuré", et la lutte anarchiste donner accès à une "solidité » comparable à «l'ordre stable» des anciennes sociétés «de bâtisseurs et de colonisateurs"?

La réponse à ces questions mettait en jeu, dans les deux cas, un antagonisme fondamental qui jouait un rôle de tout premier plan : pour Einstein comme pour Weber, la valorisation résolue de certaines modalités d'expérience collective (qui se traduisait, chez eux comme chez bien d'autres auteurs de l'époque, par 


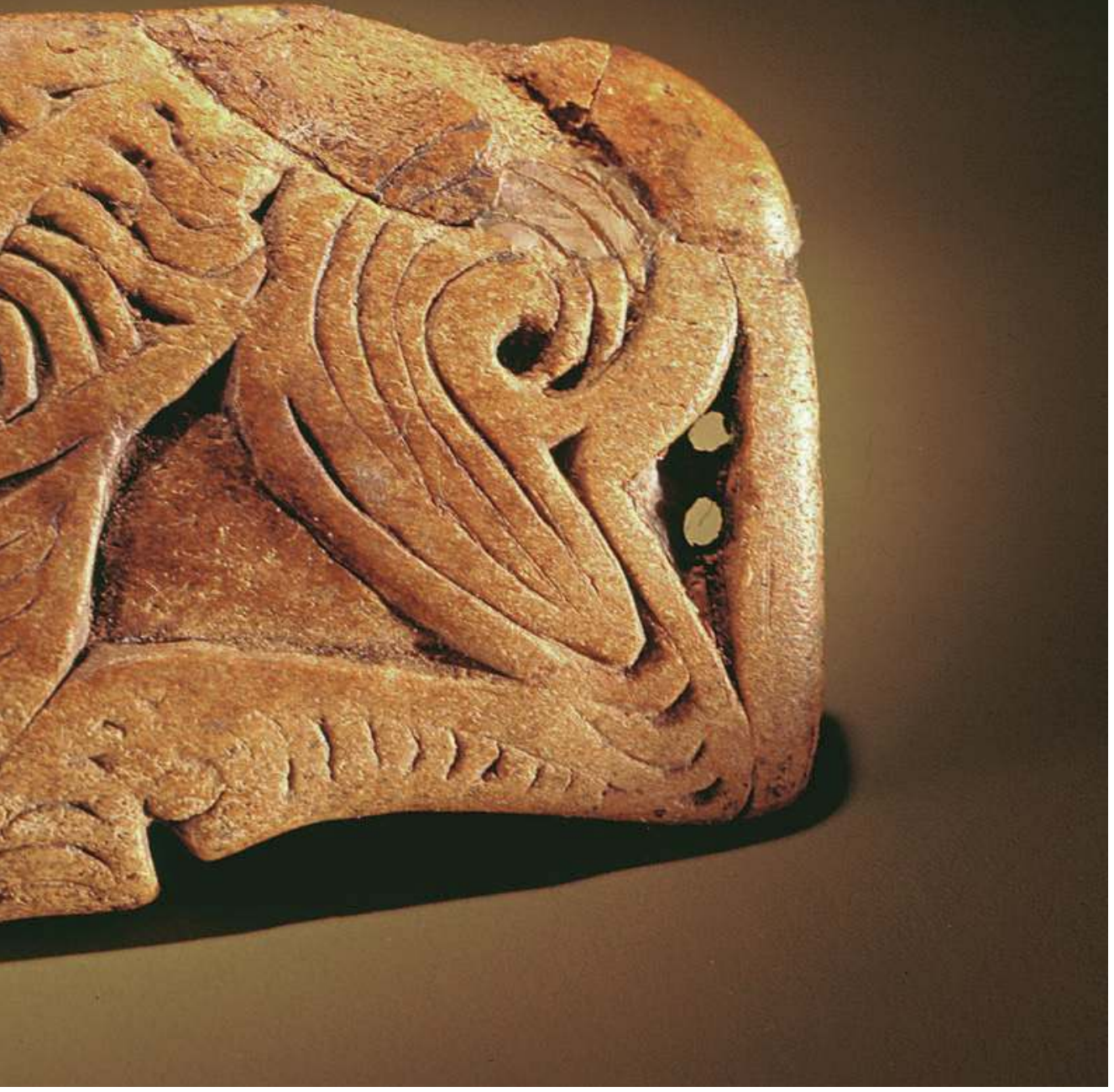

un attrait pour l'ethnologie des «sociétés secrètes") était d'abord et avant tout l'envers d'une posture qui faisait figure de repoussoir absolu et contre laquelle tous deux n'avaient pas de mots assez durs, la quête individualiste moderne de la "singularité " et la promotion du «moi». La recherche compulsive d'une originalité à tout prix, la tentative volontariste et nécessairement vouée à l'échec de construction artificielle d'une "personnalité» comptaient pour eux parmi les stéréotypes sociaux les plus exaspérants de la modernité, et ils mettaient en relation ce phénomène avec la quête éperdue d'une « autonomie » absolue de l'art qui s'épuisait dans une célébration narcissique de lui-même ${ }^{10}$. Einstein écrivait par exemple dans le texte sur les bronzes antiques :

Les critères exclusivement esthétiques ou formels traduisent précisément la pauvreté spirituelle d'une grande part des images actuelles. [...] Cet art-là [l'art de la haute Antiquité], reconnaissons-le, n'avait pas la prétention de constituer une sphère autonome à partir de tubes de peinture et de vagues esthétismes. («Exposition de statuettes de bronze antiques", infra, p. 234)

-

10. Je renvoie ici à mon article «Une note de Max Weber sur les danseuses indiennes. Arts du corps, savoir lettré, charisme héréditaire », Revue de synthèse 129, 2008. 
Dans la tripartition d'Einstein, le moment «nomade » et le troisième moment, celui de la "paraphrase", étaient ainsi identifiés à des formes de pathologie du moi : menace de «dissolution métamorphique» du moi trop passif devant ses visions dans le premier cas et, dans l'autre, hypertrophie de l'ego. La figure de Kandinsky illustrait exemplairement, dans l'analyse einsteinienne du Cavalier bleu, le basculement entre ces deux écueils qui menaçaient l'art de son temps, l'excès visionnaire et la chute dans le « décoratif ». Tels étaient les pôles de négativité auxquels Einstein opposait la positivité du moment «tectonique». Mais la question des modalités du passage à ce dernier n'est pas pour autant résolue.

\section{Le tectonique et son autorité}

La pensée d'Einstein se caractérise, comme les "métamorphoses» auxquelles elle accorde une si grande place, par une circulation continuelle entre des niveaux de signification rattachés entre eux sur un mode analogique. Entre le "psychisme » du moi, la sphère des relations sociales et politiques et le monde de l'art, Einstein postulait des correspondances, des passages qu'il effectuait sans transition parce que, pour lui, ils allaient de soi. La coutume de désigner Einstein comme un "critique d'art" fait parfois oublier que lui-même n'accordait pas de réelle prédominance à l'art sur les autres sphères que l'on vient d'énumérer. Certains textes, comme le fragment posthume "Concept et figure", évacuaient même toute référence non seulement à des œuvres d'art, mais aussi à la création artistique en général. Dans "Le Cavalier bleu ", il déclarait sans détour : "Il en allait de quelque chose de plus important que la seule peinture, à savoir d'une transformation de la structure psychique.» Il n'est pas exact de dire que plusieurs périodes se succèdent dans sa pensée, et qu'à une croyance dans le pouvoir révolutionnaire de l'art fait suite une désillusion qui aurait conduit Einstein vers l'action politique : en toute rigueur, il n'attribua à aucun moment à l'art un primat incontesté. Il affirma dans tous ses textes que l'art ne pouvait être que subordonné à d'autres fins que lui-même. C'est là une prémisse essentielle. Elle explique, pour une part, le caractère déroutant de textes sur l'art dans lesquels se donnent à voir un regard artistique souverainement clairvoyant et, tout à la fois, une manière souvent cavalière d'aborder les œuvres d'art singulières, en s'abstenant dans bien des cas de les décrire et même de les nommer.

Dans ses études sur les arts primitifs et les arts anciens, Einstein mettait en avant les fonctions rituelles et sociales des œuvres d'art; après Negerplastik surtout, il eut recours à des lectures ethnologiques pour étayer cette thèse. Les anthropologues d'aujourd'hui jugent à maints égards datées les observations ethnologiques d'Einstein. Mais elles l'étaient déjà de son vivant, et il est manifeste qu'il ne plaçait pas son ambition dans l'apport de matériaux nouveaux dans ce domaine, pas plus que dans celui de l'histoire de l'art. Son bagage ethnologique un peu sommaire jouait pour lui davantage le rôle d'une source d'inspiration poétique que d'un cadre scientifique. À aucun moment, Einstein ne prétendit être en mesure d'enrichir d'une quelconque façon un savoir spécialisé, que ce fût dans le domaine de l'art ou dans celui de l'ethnologie des cultures "primitives ». Le critère de validation auquel il était prêt à soumettre ses textes n'était pas celui-là.

Même l'objectif politique ne fut à aucun moment, pour Einstein, une finalité exclusive et inconditionnée. La preuve en est donnée, a contrario, dans le texte qu'il consacra en 1936, pendant la guerre d'Espagne, à la mémoire du «camarade
Pangi, Lega, Metropolitan

Museum of Art, The Michael C. Rockefeller Memorial

Collection, legs de Nelson

A. Rockefeller, 1979 . 


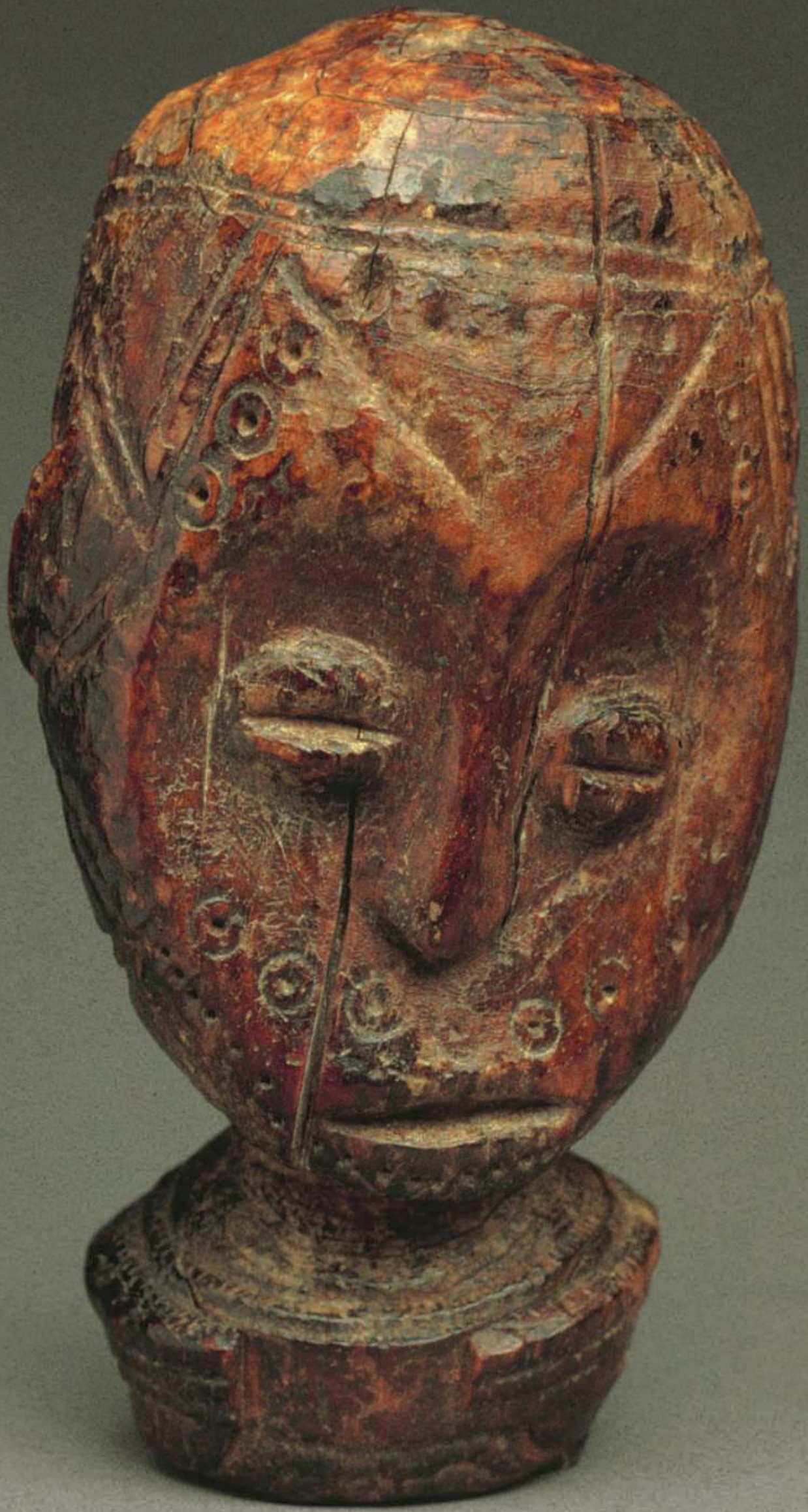




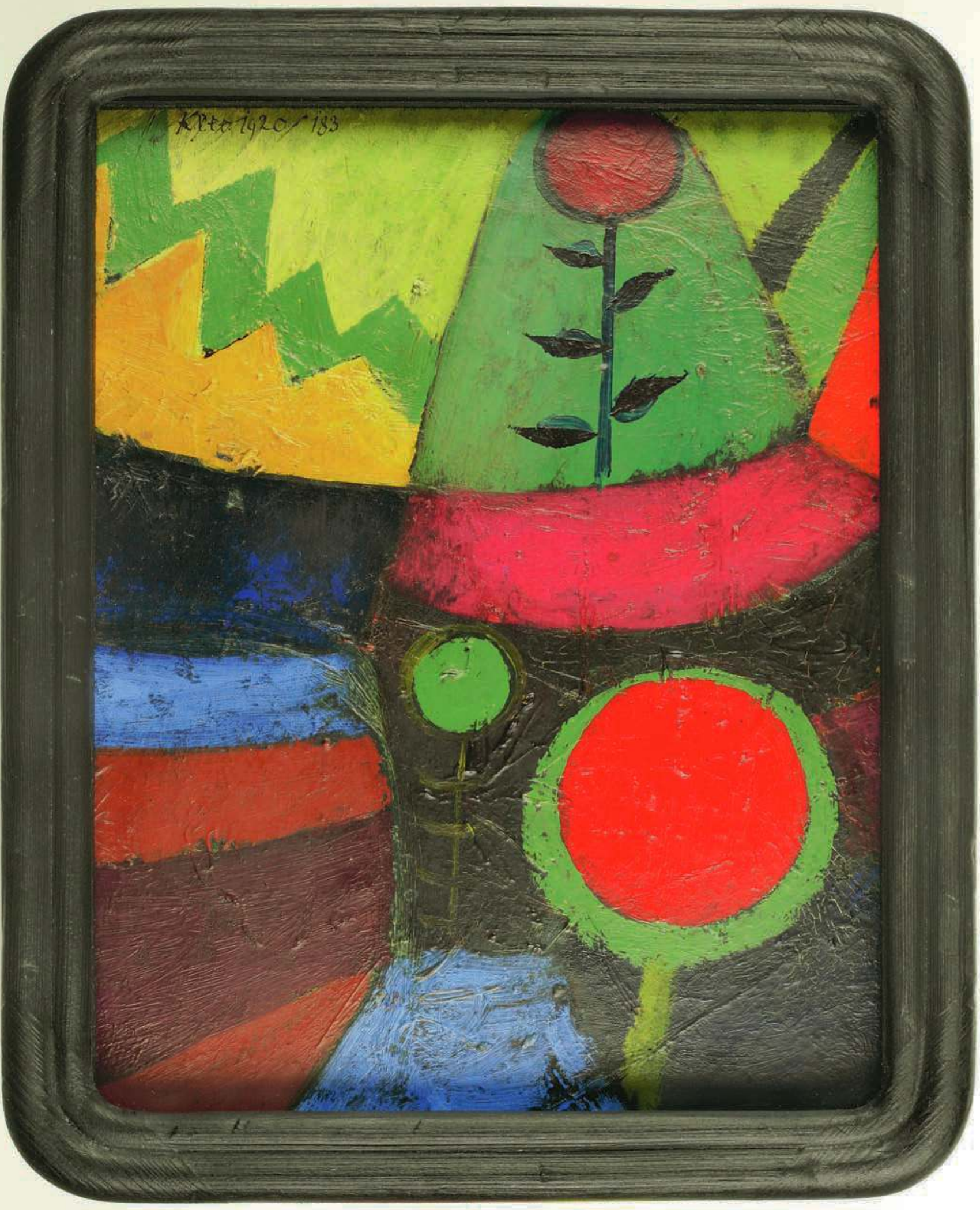

Fig. 8 Paul Klee, Drei Blumen, 1920. Zentrum Paul Klee, Berne, Livia Klee Donation. 
Durruti ». Dans cette harangue qui fit l'objet d'une diffusion radiophonique, Einstein se plie sans réserve à l'énoncé d'un discours de propagande. La rhétorique et le vocabulaire sont entièrement d'emprunt, et l'auteur renie son écriture pour laisser ce discours impersonnel parler à sa place. À y regarder de plus près, pourtant, on reconnaît sous la gangue du discours prolétarien une problématique einsteinienne qui fait écho à ses textes antérieurs. En sous-main, dans ce texte, Einstein cherche à définir les modalités d'organisation d'un collectif qui soit un équivalent moderne du moment «tectonique ». La solution réactionnaire visant à reconstituer purement et simplement une structure autoritaire n'était pas envisageable, de son point de vue : sa présence dans la «colonne Durruti" ne trouvait sa justification que dans le fossé qui séparait son engagement de la stricte allégeance au pouvoir d'un seul; la "colonne" ne devait pas se confondre avec une simple armée de soldats, la cause défendue par les camarades ne pouvait être qu'aux antipodes des buts de guerre imposés malgré eux aux combattants, et Durruti ne devait pas être regardé comme un chef militaire, fût-il charismatique. "Nous laissons volontiers aux fascistes le fétichisme du chef et la fabrication des vedettes ", écrivait-il. On peut juger ou non cette argumentation convaincante; mais elle structure tout le texte. L'autorité de l'organisation politique et de sa "cause" n'était pas justifiée par elle-même, comme elle pouvait l'être dans le «dogmatisme» et le "style dictatorial» du moment tectonique. Einstein fondait la légitimité qu'il lui reconnaissait, en fin de compte, sur l'intérêt de «l'individu " et de sa "personnalité » à la réalisation de l'idéal révolutionnaire : «Nous n'obéissons pas à des généraux mais nous poursuivons la réalisation d'un idéal social qui implique, entre autres, le développement maximal de l'individualité prolétaire. La militarisation, en revanche, a été jusqu'ici un moyen volontiers utilisé pour réduire au minimum la personnalité du prolétaire. " Sous la plume d'Einstein, ces termes se chargeaient d'un poids spécifique : ce "développement» de "l'individualité ", cet idéal d'expansion de la "personnalité » étaient, dans le contexte de la guerre d'Espagne comme dans toutes les autres périodes de son existence, les valeurs auxquelles il attachait le plus de prix. Ses textes présentent, de ce point de vue, une grande continuité, jusque dans ce moment historique dramatique.

Présente en filigrane jusque dans l'écriture contrainte de "La colonne Durruti ", la thématique du développement d'une "personnalité " qu'on pourrait appeler "supra-subjective », dépassant les frontières contingentes de l'ego individuel, était le point focal de la pensée d'Einstein. Sa quête tenace d'un moi vivant, contre les fixations de toute sorte, était marquée par la lecture de Nietzsche et n'était pas sans affinité avec des courants ultérieurs de la Lebensphilosophie, la « philosophie de la vie ». Mais il la définissait là encore d'une façon toute personnelle. La très importante lettre à Kahnweiler de juin 1923, retrouvée et publiée par Liliane Meffre, peut être lue comme le manifeste de cette pensée einsteinienne du " moi » : elle met en jeu une approche "qualitative» des catégories de l'espace et du temps (Einstein parle d'une "théorie qualitative du temps »). Le moi «fonctionnel», opposé au moi comme "substance métaphysique", se définit par la labilité de ses frontières, qui peuvent se déplacer, connaître différents mouvements d'expansion et de restriction imprévisibles. Ce moi «croît, disparaît et se complexifie exactement comme l'espace cubiste " : la "personne croît et décroît en volume, en sentiment de soi ou en sensation des choses, en tension temporelle ». En rendre compte ne consiste pas à "décrire" ni à "dépeindre " un vécu, mais à traduire ce "changement d'intensité ", ces "variations » dans le "sentiment du temps ", sa "force ", son " ampleur ", sa «signification », son « orientation" ${ }^{11}$. Einstein nommait ainsi, d'une façon aussi
11. Einstein-Kahnweiler, 1993, p. 139. 
peu rigide que possible, la qualité « d'intensité » de la sensation du moi qu'il opposait au travail de la mort et à ses déficits de circulation.

Le manuscrit posthume "Concept et figure » reprenait la tripartition qui fait l'objet de cet article en l'appliquant aux états du moi : le moment nomade correspondait à ce qu'Einstein appelait la " personne animiste complexe ", et le moment classique à ce qu'il désignait comme la "fiction de la personne fermée", associée à une division du monde en un "secteur subjectif » et un "secteur objectif ». Entre les deux, le moment tectonique était celui où se délimitait une enveloppe, une forme provisoire du moi, une identité mouvante qui n'était pas encore «fermée ». Le moi «tectonique" se situait ainsi dans un point d'équilibre instantané entre l'impersonnel et le personnel, le passif et l'actif.

Dans cette quête d'un moi vivant, l'art jouait selon Einstein un rôle majeur parce que, dans les termes de la lettre de juin 1923, "les peintres » de son temps étaient « les seuls à avoir eu du courage ». "Les travaux des "cubistes", ajoutaitil, ont été pour moi la confirmation qu'il est possible d'introduire des nuances nouvelles dans la sensation. " La littérature, "avec son lyrisme et ses petites suggestions de cinéma", était à la traîne par rapport à l'art et à la science, mais le travail de différenciation qualitative accompli par ces derniers pouvait trouver un équivalent dans la création langagière : "Il est possible non seulement de transformer la perception, mais aussi de transformer l'équivalent linguistique et les sensations ${ }^{12}$." Si les sphères de l'art, de la science, de la littérature, mais aussi de la religion et de la politique étaient en un sens placées sur le même plan et susceptibles de se répondre entre elles par des analogies, c'est parce qu'elles étaient toutes abordées par Einstein dans la perspective d'une ouverture de l'intensité du moi. Dans une étude récente sur les «métamorphoses » chez Einstein, Charlotte Weyrauch a souligné à juste titre l'importance d'une formule de Negerplastik: "Seule la forme artistique correspond à l'être des dieux. " L'affinité entre la religion et l'art tenait avant tout, dans cette optique, à "l'insatisfaction" manifestée par l'une et par l'autre à l'égard du «monde visible» et de tout «naturalisme du donné ». Certaines difficultés évoquées plus haut trouvent peut-être là une amorce de dénouement : dans la logique d'Einstein, la transition entre le mode d'appréhension des arts rituels "primitifs" et celui d'un art moderne comme le cubisme, ou encore, dans un autre domaine, entre l'organisation dictatoriale des sociétés " tectoniques » et celle de collectifs révolutionnaires comme la colonne Durruti, ne devait pas être entendue comme une articulation directe, mais plutôt comme une comparaison construite à partir du terme tiers qu'était la définition d'un «moi fonctionnel ». Lui seul, dans sa posture d'appréhension et, en l'occurrence, de renouvellement du réel et de dépassement du "déjà visible", pouvait faire le lien entre des structures aussi dissemblables, qui n'étaient pas les héritières les unes des autres et entre lesquelles n'existait aucune solution de continuité.

La définition einsteinienne du tectonique, comme nous le suggérions déjà au début de cet article, ne procède donc pas d'un désir caché de restauration d'un ordre ancien, catégorique. Sa part "minérale», engagée dans la solidité de la pierre, a pour pendant un versant plus aérien, qui trouve son expression exemplaire dans les bronzes travaillés au repoussé, ou encore dans les statuettes et les masques de bois creusé. Einstein conjugue deux définitions du tectonique comme de la sculpture : celle qui vise une forme pleine, saturée de matérialité, mais aussi, indissociablement, celle qui s'attache à la forme sculptée comme à une enveloppe, à la frontière délicate d'un espace vide. Einstein maintient 
constamment ces deux modalités, qu'il associe lui-même à une polarité masculin-féminin à travers le couple du " pilier phallique » et du " crâne ». Il ne s'arrête pas seulement à la sculpture pleine comme le faisait Herder dans sa poétique de la "plastique » comme «toucher »; il développe aussi, comme Gottfried Semper, une version spatiale du tectonique, en creux. De la même façon, chez Einstein, "l'autorité " du moment tectonique ne se déploie jamais exclusivement comme une forme d'obéissance à un ordre, mais aussi comme une maîtrise de l'ordre auquel le moi se soumet: dans les sociétés archaïques évoquées dans le texte sur les bronzes, l'ordre cosmique et divin s'exerce sous une forme "dictatoriale " mais les hommes dominent, par le tectonique, les dieux qui les dominent. Ils se donnent mutuellement vie. Et mort. Car le rapport du tectonique avec la mort est là aussi paradoxal : érigé pour célébrer la mémoire des morts, il sert aussi à les évacuer, à autoriser leur oubli. Il n'est pas un texte d'Einstein sur l'art primitif où il n'évoque la coutume de jeter les œuvres. Le moment tectonique se situe dans cette polarité, comme le résume cette réflexion sur l'art africain : "L'art africain meurt avec la religion africaine et, au demeurant, celle-ci contient déjà les germes d'une décadence. L'esprit du mort n'a peut-être pas toujours besoin de la statue. Il peut établir sa demeure dans n'importe quelle plante ou n'importe où ailleurs. Ce n'est pas tout : l'Africain aime oublier les ancêtres qui l'assaillent. Il les fuit dans l'exogamie. [...] Peu à peu, la force magique de ces sculptures s'épuise et elles sont oubliées. Ces raisons ont aussi contribué à l'immense décadence de ce grand art que fut l'art africain. » ("Art exotique»)

CNRS/ENS, Laboratoire Pays Germaniques ikalinowski@free.fr

mots clés / keywords : Carl Einstein // Carl Einstein • sculpture // sculpture • nomade // nomadic - tectonique // tectonic $\cdot$ classique // classical $\cdot$ style archaïque // archaic style.

\section{Résumé / Abstract}

Isabelle Kalinowski, Les trois moments de Carl Einstein - En accordant une place nouvelle à des textes encore mal connus de Carl Einstein, traduits dans ce numéro, qui font le lien entre sa réflexion sur les arts primitifs et son intérêt pour des styles « archaïques », comme «L'art des nomades d'Asie centrale » (1931) ou «Exposition de statuettes de bronze antiques» (1933), l'article reconstitue une tripartition cyclique qui ponctue la vision einsteinienne des arts, des sociétés et du psychisme humain. Einstein oppose à un moment «tectonique » de stabilisation provisoire et collective d'un sens un moment «classique » dans lequel celui-ci se routinise et un moment «nomade 》 qui recueille l'héritage de classicismes oubliés, sur un mode conservateur, mais aussi novateur, car il les réinvente en les déplaçant. Einstein ne perd jamais de vue la circulation entre ces différents moments, même lorsqu'il semble, à certaines étapes de son parcours personnel, en privilégier un aux dépens des autres.
Isabelle Kalinowski, Carl Einstein's Three Moments - This paper sheds a new light on writings by Carl Einstein which are still little known and which are translated in this volume. In these texts, such as "Nomad Art from Central Asia" (1931) and "Exhibition of Bronze Statuettes B.C." (1933), Einstein's reflection on primitive arts meets his interest for "archaic" styles. This paper restores the cyclic sequence of three moments which characterizes Einstein's understanding of arts, societies and human psyche. Einstein distinguishes between the "tectonic" moment when the sense is temporarily and collectively stabilized, the "classical" moment of its routinization, and the "nomadic" moment when lost classicisms are inherited on a conservative yet innovative mode, for they become reinvented through their displacement. Einstein has always in mind the fact that these moments are interrelated, even though, at some points during his career, he seems to give priority to one or another. 